\title{
Ion-water coupling controls class A GPCR signal transduction pathways
}

\author{
Neil J. Thomson ${ }^{1}$, Owen N. Vickery ${ }^{1,2}$, Callum M. Ives ${ }^{1}$, and Ulrich Zachariae ${ }^{1, \mathbb{W}}$ \\ ${ }^{1}$ Computational Biology, School of Life Sciences, University of Dundee, Dundee DD1 5EH, UK., \\ ${ }^{2}$ Current address: School of Life Sciences, University of Warwick, Coventry CV4 7AL, UK.
}

\begin{abstract}
G-protein-coupled receptors (GPCRs) transmit signals across the cell membrane, forming the largest family of membrane proteins in humans. Most GPCRs activate through an evolutionarily conserved mechanism, which involves reorientation of helices and key residues, rearrangement of a hydrogen bonding network mediated by water molecules, and the expulsion of a sodium ion from a protonatable binding site. However, how these components interplay to engage the signal effector binding site remains elusive. Here, we applied information theory to molecular dynamics simulations of pharmaceutically important GPCRs to trace concerted conformational variations across the receptors. We discovered a conserved communication pathway that includes protein residues and cofactors and enables the exchange of information between the extracellular sodium binding site and the intracellular G-protein binding region, coupling the most highly conserved protonatable residues at long distance. Reorientation of internal water molecules was found to be essential for signal transmission along this pathway. By inhibiting protonation, sodium decoupled this connectivity, identifying the ion as a master switch that determines the receptors' ability to move towards active conformations.
\end{abstract}

Correspondence: u.zachariae@dundee.ac.uk

\section{Introduction}

G-protein coupled receptors (GPCRs) form the largest family of cell surface receptor proteins in humans with over 800 members. Spanning the plasma membrane, GPCRs act as signal transducers to enable transmembrane communication into the cell. Extracellular ligand binding leads to conformational changes in the receptors that expose effector protein binding sites on their intracellular face. The effectors, including hetero-trimeric G-proteins and $\beta$-arrestins, initiate a spectrum of intracellular signalling cascades that cause a variety of physiological changes (1). As such, GPCRs form the main target for drug therapies, with over $30 \%$ of US food and drug administration (FDA) approved drugs targeting about 108 different GPCRs (2).

Class A receptors comprise the vast majority of GPCRs. High-resolution crystal structures of class A GPCRs in the inactive state show a sodium $\left(\mathrm{Na}^{+}\right)$ion bound to $\mathrm{Asp}^{2.50}$ (Ballesteros-Weinstein numbering system), a highly conserved, ionizable residue central to their transmembrane domain (Fig. 1). By contrast, structures of receptor active states reveal a collapsed sodium binding site, unable to accommodate the ion $(3,4)$. Consequently, a wide range of studies have suggested a role for sodium in GPCR activation (4$10)$. The absence of sodium is likely to trigger protonation of $\operatorname{Asp}^{2.50}(6,11,12)$, while the sodium binding site is further embedded in a conserved network of water molecules that connect polar residues and undergo significant re-positioning

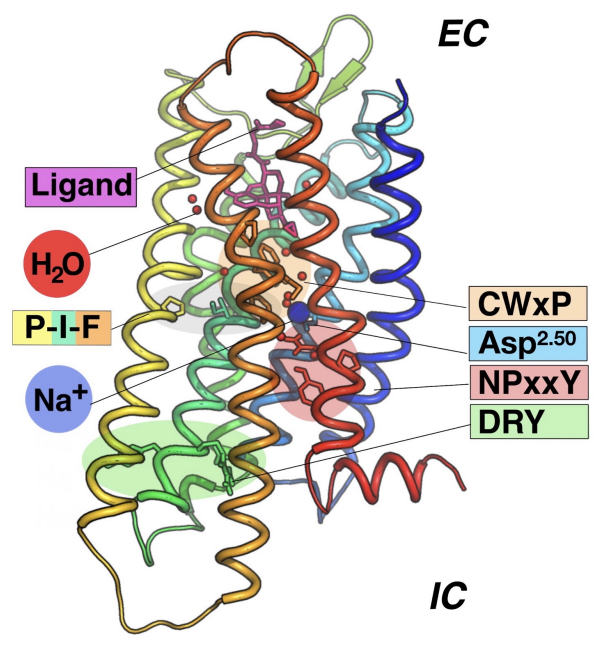

Fig. 1. Key class A GPCR features and microswitches. Shown are the ligand binding pocket, internal waters, sodium ion $\left(\mathrm{Na}^{+}\right)$, conserved microswitch motifs, and the primary sodium binding residue $\mathrm{Asp}^{2.50}$ in the $\mu$-opioid receptor (pdb: $4 \mathrm{dkl}$ ) as example. EC and IC denote the extracellular and intracellular side, respectively. Important microswitch motifs include the CWxP motif at the ligand binding site, linked directly to the sodium ion binding pocket, and the P-I-F motif, which is strongly coupled to the presence of ligands (23). The NPxxY ${ }^{7.53}$ motif is located proximal to the ion binding site and includes $\mathrm{Tyr}^{7.53}$, which can gate a transmembrane water channel according to previous simulation studies $(12,15)$.

upon activation (13-15).

Transitions between distinct rotameric conformations of evolutionarily conserved residues, termed microswitches, are believed to underpin the large scale conformational changes of transmembrane helices that govern activation $(1,13,16-23)$. Many microswitches form part of the interconnected polar network. Amongst these residues, the ionizable DRY motif at the intracellular face plays a key role in G-protein binding, and forms ionic locks that maintain the inactive receptor state $(1,20,24)$ (Fig. 1).

These observations suggest that the ion binding site, protonation, water-mediated interactions, and conserved residues act together to affect G-protein signalling. However so far, the functional interaction of these elements remains insufficiently understood $(4,14,25)$. Here, we hypothesized that these components communicate state changes with each other to induce the global conformational changes determining receptor activation.

GPCR signal transduction is a process whereby information (i.e. about a ligand binding event) is sent, encoded, transmitted and received, in parallel with the tenets of an information system (26). Therefore, $\mathrm{Na}^{+}$, water, and microswitches can be seen to act as essential elements of an information system. The function of water molecules in GPCRs has so far predominantly been studied from a structural perspective $(14,27)$. To resolve their role in signalling, we applied 
Shannon's mutual information $(26,28)$ and McGill's interaction information (also called co-information) (29-32), as "State Specific Information" (SSI), in order to quantify information shared through coupled transitions of discrete residue and internal water states. In contrast to information theoretic approaches that analyze correlations in protein dynamics in cartesian space (most often $\mathrm{C}_{\alpha}$ motions) $(6,31,33-$ 37), SSI derives from internal coordinate and state-based mutual information frameworks (38-41). It is therefore closely aligned with the concept of GPCR microswitches by requiring a change between at least two distinct conformational states for information exchange.

By combining two independent molecular dynamics simulations with ensembles which differ only in the condition of a specific component, e.g. whether Asp ${ }^{2.50}$ binds a sodium ion, the combined ensemble reflect the effects of that change (Fig. 2). Similar to the Jensen-Shannon divergence $(42,43)$, SSI then quantifies information shared from that components change in state caused by the transition between individual ensembles and the resulting coupled changes in internal residues and water states in the combined ensemble. As a consequence, SSI establishes causality by tracing the information transfer back to a root cause (see also Materials and Methods).

Accordingly, we conducted all-atom molecular dynamics simulations initiated from three inactive-state, antagonistbound class A GPCRs: the $\mathrm{A}_{2 \mathrm{~A}}$-adenosine receptor ( $\left.\mathrm{A}_{2 \mathrm{~A}} \mathrm{AR}\right)$, the $\mu$-opioid receptor $(\mu \mathrm{OR})$, and the $\delta$-opioid receptor $(\delta \mathrm{OR})$. For each receptor, independent molecular dynamics simulation data of $1.7 \mu$ s length was collected, performed in three different receptor states of the main ion binding residue: $\mathrm{Asp}^{2.50}$ in a charged state with sodium bound $\left(\mathrm{Na}^{+} / \mathrm{D}^{-}\right)$, Asp 2.50 in a charged state with sodium removed $\left(\mathrm{D}^{-}\right)$, and $\mathrm{Asp}^{2.50}$ in a protonated state without sodium $\left(D^{0}\right)$. Transitions between states were represented by pairwise combinations of independent simulations in the respective states (Fig. 2) and analyzed using SSI. Thereby, we extracted information from persistent changes in internal residue and water states occurring in all three receptor types that are coupled specifically to sodium binding or protonation of Asp 2.50 .

Our results reveal how ion binding and $\mathrm{Asp}^{2.50}$-protonation are coupled to the dynamics of internal water molecules and protein microswitch conformations. Altogether, these elements form a long-range information transfer pathway between two highly conserved ionizable regions - Asp ${ }^{2.50}$ and the intracellular DRY motif at the G-protein binding site. By determining Asp ${ }^{2.50}$-protonation, sodium binding is found to act as the master switch which inhibits concerted transitions towards active state conformations throughout the receptor.

\section{Results}

We first monitored the water occupancy of protein cavities. Five pockets, located in receptor regions of highly conserved motifs, exhibited large probability densities for internal water in all simulations (Fig. 3A,C). Four of these sites are conserved in class A GPCRs (14). Information transferred across

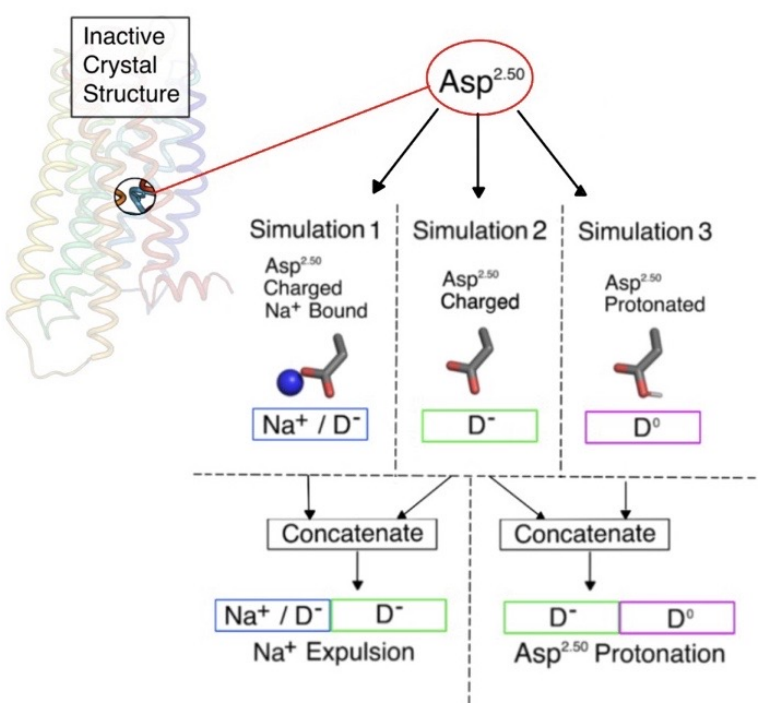

Fig. 2. Molecular dynamics simulation approach. For each of the three class $A$ GPCRs, three independent simulations with unique $\mathrm{Asp}^{2.50}$ and ion binding states were initiated from the inactive crystal structure: $\mathrm{Asp}^{2.50}$ charged with sodium bound $\left(\mathrm{Na}^{+} / \mathrm{D}^{-}\right)$, Asp ${ }^{2.50}$ charged without sodium bound $\left(\mathrm{D}^{-}\right)$, and $\mathrm{Asp}^{2.50}$ protonated without sodium $\left(\mathrm{D}^{0}\right)$. Receptor transitions caused by expelling sodium, and subsequently protonating $\mathrm{Asp}^{2.50}$, were represented by concatenating two independent trajectories into one large trajectory. This reflects a binary change to the state of the focal site, corresponding to 1 bit of information (e.g. 'protonated or de-protonated'), which acts as the source of information.

the water sites was calculated based on pocket occupancy as well as water orientation. We then investigated information exchanged by coupled conformational state transitions as SSI between 7875 residue and internal water pairs for each of the three receptors. The sodium binding and protonation site was treated as an independent binary residue microswitch (the information source, see Fig. 2) To elucidate pathways conserved across all three receptors, we determined the geometric mean of results from all common residue BallesterosWeinstein positions, totaling 121 residues.

\section{An information transfer pathway from the $\mathrm{Na}^{+}$pocket to the intracellular G-protein binding interface. Rather} than affecting the entire protein, SSI was shared between a small group of key microswitch and water pairs, both for sodium expulsion and $\mathrm{Asp}^{2.50}$-protonation ( $\sim 5 \%$ and $6 \%$, respectively). Within this core group, our analysis revealed long-range information transfer sent from the ion binding site to the P-I-F motif, the NPxxY motif, and the distal ionizable DRY motif involved in the G-protein binding interface (Fig. 3B). We found that the conformation of residue $\mathrm{Arg}^{3.50}$ of the $\mathrm{DR}^{3.50} \mathrm{Y}$ motif, at a distance of more than $20 \AA$, was strongly impacted by $\mathrm{Asp}^{2.50}$-protonation (Fig. 3B).

Internal water molecules acted as important players in the transmission of information emerging from the ion binding site. The conformations of the water molecules were more indicative of the state of the ion binding site than the majority of the protein residues (Fig. 3B). This suggests that water molecules are an integral part of the signal transduction mechanism, and that the internal water molecules form additional elements in an extended set of microswitches. Furthermore, it is known that water 1 (Wat1) forms interactions 
present in the inactive receptor state, while interactions with water 2 (Wat2) are correlated with the active receptor state (14). In agreement with this, protonation of $\mathrm{Asp}^{2.50}$, which has been linked to activation (12), diminished the occupancy of site Wat1 by $\sim 25 \%$, whereas it increased Wat 2 occupancy by $\sim 25 \%$.

The magnitude of information received from the ion binding site across all three receptors, projected onto the $\mu \mathrm{OR}$ crystal structure, reveals a pathway that includes water and transmits information between Asp ${ }^{2.50}$ and the DRY motif (Fig. 3C). Information travels in the form of propagated conformational changes along helix 7 and across the receptor base. The tight connectivity of coupled transitions on this route allows changes at the ion binding site to affect the conformation of the distal G-protein binding site, establishing long-range coupling between the ionizable residues $\mathrm{Asp}^{2.50} \mathrm{Asp}^{3.49}$, and $\mathrm{Arg}^{3.50}$. The ability of these residues to change protonation state has previously been suggested to be a hallmark of class A GPCR activation (24). Following protonation of $\mathrm{Asp}^{2.50}$, the conformational change at $\mathrm{Arg}^{3.50}$ also impacted the conformation of residue $\mathrm{X}^{6.30}$ (the helix 6 ionic lock), and position $\mathrm{X}^{34.57}$ on intracellular loop 2 (ICL2), which is known to play a role in effector protein binding specificity and bias (44-46). Taken together, these results therefore show that the sodium binding pocket communicates at long-range with key interfacial residues that are crucial for effector protein interaction, receptor activation and signal bias.

Efficient information transfer requires coupling of Asp ${ }^{2.50}$-protonation to water. We found that two key motifs implied in receptor activation, the NPxxY and the DRY motif, became conformationally coupled after Asp ${ }^{2.50}$ protonation (Figs. 3B; for full SSI data see Supplementary Information). Unlike sodium expulsion, $\mathrm{Asp}^{2.50}$-protonation caused all residues of the $\mathrm{N}^{7.49} \mathrm{PxxY}$ motif to undergo various degrees of conformational rearrangement. Structurally, $\mathrm{Asp}^{2.50}$-protonation directly altered the Asp ${ }^{2.50}-\mathrm{Asn}^{7.49}$ hydrogen bonding pattern, the conformation of the hydrophobic barrier created by $\operatorname{Tyr}^{7.53}$ (12), and the orientation of water molecules Wat1 and Wat2, proximal to the NPxxY motif (Fig. 3B).

To quantify the functional effect of the intercalated water molecules Wat1-Wat5 (Fig. 3A,C) on coupled conformational changes, we calculated the co-information parameter (co-SSI, see Materials and Methods for details) (29-32). Here, positive or negative co-SSI values indicate that the coupling between the ion binding site and each microswitch is stabilised or destabilised, respectively, by their interaction with water molecules. This is equivalent to water amplifying or attenuating the shared information.

We observed that the water network had a particularly pronounced effect on the information pathway upon $\mathrm{Asp}^{2.50}$ protonation (Fig. 4A,B). Specifically, reorientation of Wat1 and Wat2 was critical to establish communication between the NPxxY and DRY motifs. It also stabilized conformational changes along the pathway to the G-protein binding site as a whole, amplifying the majority of SSI transfer from the protonation site to the microswitches.
The negative value of co-information between Pro ${ }^{7.50}$, Wat1, and the $\mathrm{Asp}^{2.50}$-protonation state, by contrast, implies that reorientation of Wat 1 destabilises the conformational state of Pro ${ }^{7.50}$. This may contribute to the kinking of helix 7 seen upon activation (for full co-SSI data see Supplementary Information).

\section{Molecular mechanism of information transfer propa- gating from the ion binding site. To elucidate how infor-} mation transfer is initiated by the protein and water on the molecular level, we next analyzed the major conformational changes caused by $\mathrm{Asp}^{2.50}$-protonation. In all simulations of receptors in the $\mathrm{Asp}^{2.50}$-deprotonated state $\left(\mathrm{Na}^{+} / \mathrm{D}^{-}\right.$and $\left.\mathrm{D}^{-}\right)$, Wat 1 acted as a $\mathrm{H}$-bond donor with the side chain oxygen atoms of $\mathrm{Asp}^{2.50}$ and $\mathrm{Asn}^{1.50}$, and as a H-bond acceptor with $\mathrm{Tyr}^{7.53}$, where the Tyr $\mathrm{OH}$ group pointed towards the Wat 1 pocket (Fig. 4C).

By contrast, Asp ${ }^{2.50}$-protonation triggered substantial $\mathrm{H}$ bonding rearrangements in all three receptor types, as Wat1 rotated to accept a H-bond from the protonated $\mathrm{Asp}^{2.50}$ side chain. Furthermore, the water bridge between $\mathrm{Asp}^{2.50}$ and $\mathrm{Tyr}^{7.53}$ was fully disrupted (Fig. 4D). Thereby, $\mathrm{Tyr}^{7.53}$ flipped into its downward state in the protonated $\mathrm{A}_{2 \mathrm{~A}} \mathrm{AR}$ and $\mu \mathrm{OR}$ (Fig. 4C). This downward movement has previously been shown to correlate with the opening of a hydrophobic gate upon receptor activation, forming a continuous pore through the receptor $(12,15))$. In the $\delta \mathrm{OR}$, an identical transition was observed upon further protonation of $\mathrm{Asp}^{3.49}\left(\mathrm{D}^{3.49} \mathrm{RY}\right.$ motif), a residue that is coupled to $\mathrm{Asp}^{2.50}$-protonation through the information pathway.

Co-SSI indicated that protonation-dependent reorientation of Wat1 was essential for altering the hydrogen bonding pattern that enabled the $\mathrm{Tyr}^{7.53}$ downward swing (Fig. 4B), initiating communication with the DRY motif. In the $\mu \mathrm{OR}$, this resulted in an upward movement of $\mathrm{Arg}^{3.50}$ into its active, Gprotein binding conformation (Fig. 4C). In addition, the interhydroxyl distance between $\mathrm{Tyr}^{7.53}$ and $\mathrm{Tyr}^{5.58}$ was reduced in the $\mu \mathrm{OR}$ and $\mathrm{A}_{2 \mathrm{~A}} \mathrm{AR}$. In the $\mu \mathrm{OR}$, $\mathrm{Asp}^{2.50}$-protonation was thereby sufficient to decrease the $\mathrm{Tyr}^{7.53}-\mathrm{Tyr}^{5.58}$ interhydroxyl distance to the distribution seen in the active state, when compared to data from a $1.7-\mu$ s simulation of the active crystal structure (pdb: 6ddf) (Fig. S3). We also found that the G-protein binding cavity opened upon further protonation of $\mathrm{Asp}^{3.49}$ in the $\delta \mathrm{OR}$, as measured by the distance between $\mathrm{Thr}^{2.39}-\mathrm{C} \alpha$ and $\mathrm{Ile}^{6.33}-\mathrm{C} \alpha$ (Fig. S4). These results indicate that the protonation of two conserved ionizable residues connected by the information pathway, $\mathrm{Asp}^{2.50}$ and $\mathrm{Asp}^{3.49}$, may play a direct role in receptor activation and opening of the G-protein binding cleft.

Sodium restrains key microswitches to a single conformation. The sodium-bound form of class A GPCRs is known to be associated with their inactive state $(3,4,7$, 12). Notably, three residues around the sodium binding site known to form a hydrogen bonding triplet with Wat1, $\mathrm{Asp}^{2.50}$, Tyr $^{7.53}$ and $\mathrm{Asn}^{1.50}$ (a 100\% conserved residue in class A GPCRs), were restrained to highly ordered single conformations in all three sodium-bound receptors. This was 
bioRxiv preprint doi: https://doi.org/10.1101/2020.08.28.271510; this version posted January 5, 2021. The copyright holder for this preprint (which was not certified by peer review) is the author/funder, who has granted bioRxiv a license to display the preprint in perpetuity. It is made available under aCC-BY 4.0 International license.
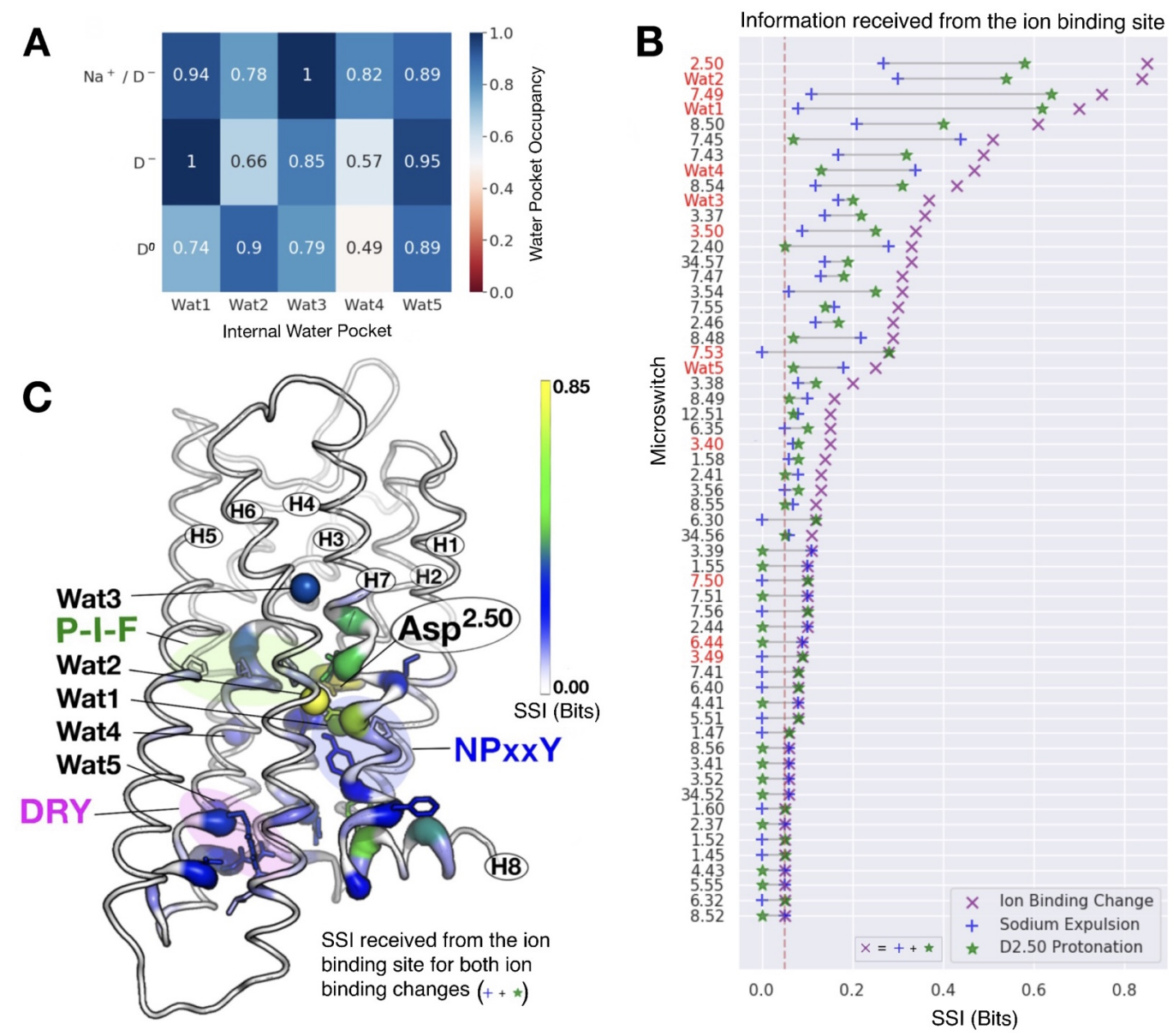

Fig. 3. State Specific Information (SSI) reveals information flow propagating through the receptor. (A) Water pocket occupancy averaged across all three receptors throughout the simulations; a value of 1 reflects complete occupancy in all receptors over the entire simulated time. (B) Information sent from the ion binding site to protein microswitches and internal water molecules (SSI) in the form of coupled conformational transitions. Shown are all significant shared SSI values in bits for sodium expulsion and $\mathrm{Asp}^{2.50}$-protonation (each corresponding to 1 bit of source information). The significance threshold is depicted by a vertical red dashed line ( 0.05 bits), and internal waters, key residues and motifs are highlighted in red. High SSI values reflect a strong coupling between the binary state change of the ion binding site and the conformational state change of a microswitch or water. (C) Information flow from the ion binding site projected as colour-code (from white, 0 bits to yellow, 0.85 bits SSI) onto the $\mu$ OR crystal structure (pdb:4dkl). Key microswitches are shown as sticks, water molecules as spheres. Microswitches involved in SSI transfer form a pathway bridging the ion binding site and the DRY motif at the G-protein binding site.

reflected by their zero conformational state entropies (see Materials and Methods) (Fig. 5). The water-mediated locking of $\mathrm{Tyr}^{7.53}$ in one single conformation, which was fully released only upon Asp ${ }^{2.50}$-protonation (Fig. 4D), prohibited $\mathrm{Tyr}^{7.53}$ from coupling its dynamics to other residues and thereby sharing information.

Additionally, Pro ${ }^{5.50}$ of the $\mathrm{P}^{5.50}$-I-F motif exhibited zero entropy, i.e. confinement to a single conformation, for the sodium-bound state. As opposed to protonation, SSI further indicated that sodium expulsion exerted an influence on both $\mathrm{I}^{3.40}$ and $\mathrm{F}^{6.44}$ of this motif. This highlights that sodium interacts with the entire P-I-F motif, which is known to be strongly coupled to ligand binding (23), as $\mathrm{F}^{6.44}$ directly interacts with $\mathrm{W}^{6.48}$, forming the base of the extracellular ligand binding pocket (47). By contrast, all microswitches displayed fluctuations between various different conformational states in the sodium-free $\left(\mathrm{D}^{-}\right)$simulations. In the protonated Asp ${ }^{2.50}$ state, only Asn ${ }^{1.50}$ was restrained to a single conformation in its vicinity.

In summary, we conclude that the sodium ion holds the receptor in the inactive state by restraining the key microswitches
$\mathrm{Asp}^{2.50}, \mathrm{Tyr}^{7.53}, \mathrm{Asn}^{1.50}$, and Pro ${ }^{5.50}$ to single conformations. As they are locked in specific states, these microswitches are unable to participate in information transfer, disconnecting the information transfer route across the receptors that emerges fully only upon Asp ${ }^{2.50}$ protonation. Since sodium dissociation is strongly coupled to Asp ${ }^{2.50}$ protonation (12), the sodium-Asp ${ }^{2.50}$ pair forms a single interlinked switch, governing information flow in GPCRs.

\section{Discussion}

Ions play important roles in GPCR signal transduction, affecting both their signal bias (48) and receptor activation $(3,4,6,12)$. In particular, a sodium ion bound to a highly conserved ionizable binding site $(3,6,7,11,24)$ is known to play a key part in activating class A GPCRs. Signal transmission is further thought to be underpinned by a tightly connected network of polar residues and internal water molecules that extends across the receptors $(14,17)$ and by the conformational changes of protein microswitches $(1,16,23)$. How these components interact to affect acti- 

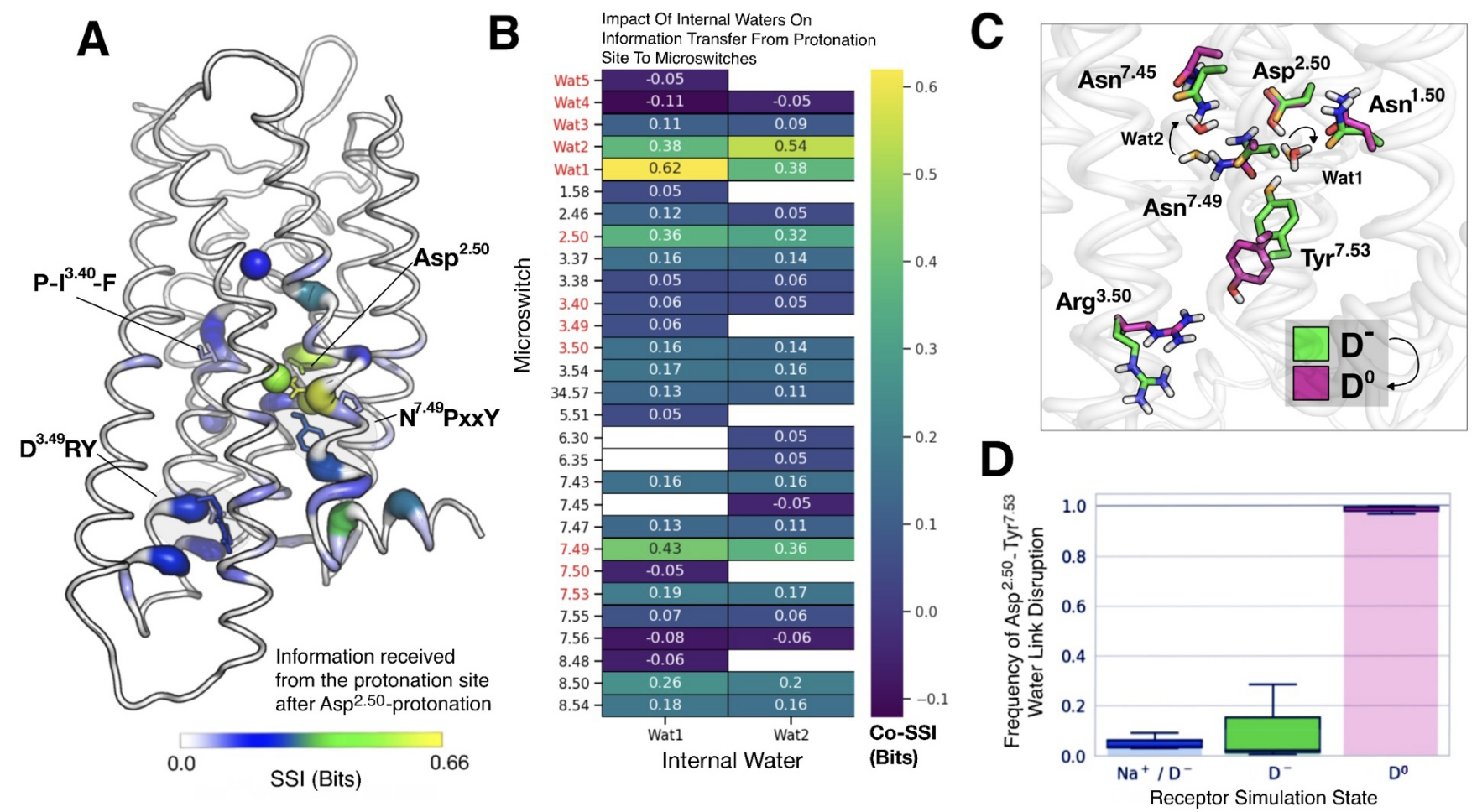

Fig. 4. Water molecules are crucial to establish communication between NPxxY and DRY motifs upon Asp ${ }^{2.50}$-protonation. (A) Information received by all three receptors after $\mathrm{Asp}^{2.50}$-protonation (SSI) projected onto the $\mu \mathrm{OR}$ structure (pdb: $4 \mathrm{dkl}$ ). (B) Impact of the two water molecules, Wat1 and Wat2, on information transfer to the microswitches (co-SSI). Microswitch motifs and internal waters are highlighted in red. Co-SSI equals SSI when two out of three components are identical (e.g., Wat2, Wat2). (C) Exemplar frames from molecular dynamics simulations of $\mu \mathrm{OR}$ in states $\mathrm{D}^{-}$(Asp ${ }^{2.50}$ charged, side chain carbon atoms in green and oxygen atoms in orange) and $D^{0}$ (neutral Asp ${ }^{2.50}$, carbon in magenta and oxygen in red). (D) Frequency of disruption of the Asp ${ }^{2.50}-$ Tyr $^{7.53}$ water bridge, averaged across the three receptors and normalised (box: dataset quartiles; whiskers: maximum and minimum disruption frequency of the three receptors). Asp ${ }^{2.50}$-protonation disrupts the water bridge, enabling coupling between $\mathrm{Tyr}^{7.53}$ and $\mathrm{Arg}^{3.50}$.

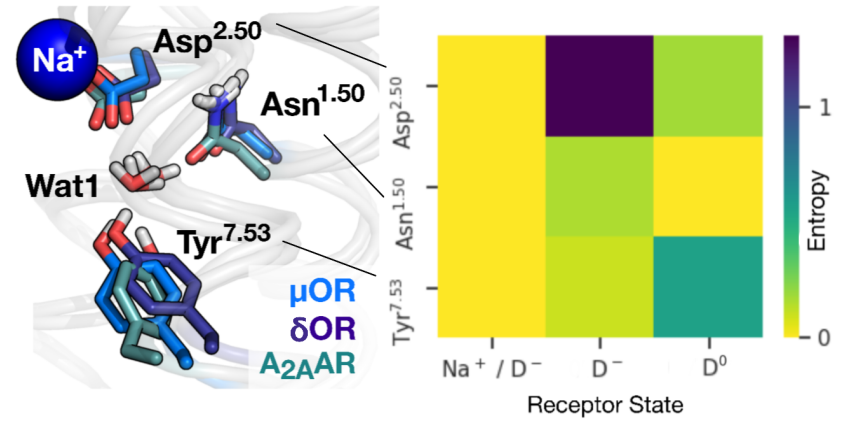

Fig. 5. Sodium confines Asp ${ }^{2.50}$, Asn ${ }^{1.50}$ and $\mathrm{Tyr}^{7.53}$ to single conformations, inhibiting communication among residue networks. Exemplar frames from molecular dynamics simulations of $\mu \mathrm{OR}, \delta \mathrm{OR}$ and $\mathrm{A}_{2 \mathrm{~A}} \mathrm{AR}$ in the $\mathrm{Na}^{+} / \mathrm{D}^{-}$simulation state (left). The side chain conformational state entropy (right) of the sodiumbound conformations of $\mathrm{Asp}^{2.50}, \mathrm{Asn}^{1.50}$ and $\mathrm{Tyr}^{7.53}$ is decreased to zero in all three receptors, fixing each residue to a specific conformation. vation and signaling, however, has been unclear. Here, we applied information theory to $\mu$ s-timescale simulations of three class A GPCRs to identify coupled transitions between these elements and examine their relation to receptor activation. Our results revealed that a long-range information transfer pathway connects the most highly-conserved protonatable residues in class A GPCRs (Asp ${ }^{2.50}, \mathrm{Arg}^{3.50}$ and $\mathrm{Asp}^{3.49}$ ) from the extracellular ion binding site to the intracellular G-protein interface (Fig. 6). The connectivity was established by protonation of Asp ${ }^{2.50}$, triggered by the removal of a sodium ion that is known to bind to $\mathrm{Asp}^{2.50}$ in the inactive receptor state $(1,4)$.

A network of ionizable residues extending through GPCRs is thought to be involved in GPCR signal transduction and may represent an evolutionary link to microbial rhodopsins $(9,12,24,49,50)$. Our findings therefore suggest that the long-range information pathway we identified may couple the protonation states of the distal conserved $\mathrm{Asp}^{2.50}$ and $\operatorname{Arg}^{3.50} / \mathrm{Asp}^{3.49}$.

In the sodium-independent, visual class A GPCR rhodopsin, two protonation switches are known to initiate activation. The first protonation event is associated with the lightinduced transition of the retinal Schiff base, central to the receptor similar to $\mathrm{Asp}^{2.50}$ in non-visual receptors, while the additional protonation occurs at the $\mathrm{D}^{3.49} \mathrm{RY}$ motif $(51,52)$. The coordinated action of such a conserved twin-protonation switch during activation of class A GPCRs would likely re- 


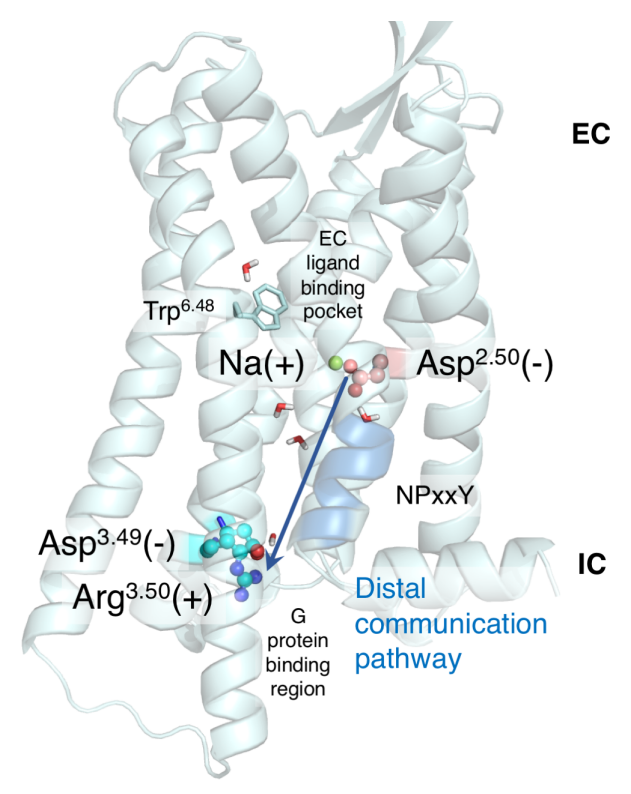

Fig. 6. A long-range information transfer pathway connects the conserved ionizable residues $\mathrm{Asp}^{2.50}, \mathrm{Arg}^{3.50}$ and $\mathrm{Asp} \mathrm{p}^{3.49}$, from the extracellular ion binding site to the intracellular G-protein interface. This allows protonatable sites at the G-protein interface regions to receive information about the $\mathrm{Asp}^{2.50}$ protonation state, and implicates $\mathrm{Asp}^{2.50}$ protonation in G-protein binding. EC, extracellular; IC, intracellular.

quire a mechanism to relay protons, and exchange information about the states of the two distal sites. The information pathway we found between $\mathrm{Asp}^{2.50}$ and $\mathrm{Asp}^{3.49}$ is composed of polarizable water molecules and residues with conformations that couple to hydrogen bonding patterns at $\mathrm{Asp}^{2.50}$, and therefore well suited to underpin proton transfer.

In many cases, ligand binding alone is thought to provide insufficient energy for receptors to transition between inactive and active conformations $(3,9)$. However, GPCRs are able to harness energy from the membrane potential gradient, potentially through ion and proton transfer $(9,12,50,53)$. Even in the presence of bound antagonists, we observed conformational rearrangements within highly conserved microswitches in the DRY and NPxxY motifs upon protonation of three receptors, which were reminiscent of the transition between inactive and active state crystal structures. This stochastic, ligand-independent sampling of active state conformations is in line with reports that suggest spontaneous $\mathrm{Asp}^{2.50}$-protonation and egress of the sodium ion may be linked to basal signalling (12), as well as the observation that active state structures exhibit no bound sodium (4). Our results suggest that sodium unbinding and protonation events, potentially coordinated between multiple conserved protonatable sites, play a key role in the activation process of class A receptors.

Two water molecules, structurally conserved across both active and inactive receptor states, are known to facilitate watermediated interactions that correlate with the inactive (water 1) and active (water 2 ) receptor states (14). We found that these two water molecules function as microswitches which couple to the ion binding states of $\mathrm{Asp}^{2.50}$. Both waters are essential for the transmission of information along a conserved pathway between the ion binding site and the DRY motif. Other water molecules play further important roles in connecting protein microswitch states over long-ranges. By disabling protonation and tightly restraining the conformation of $\mathrm{Asp}^{2.50}$ and neighboring residues and waters, sodium stabilised the inactive state of the receptor and disconnected these information transfer pathways.

Only a small fraction of class A GPCRs do not possess a sodium binding site in their transmembrane domain (for example the NK1 neurokinin receptor $(4,54)$ ), however they contain a similarly dense internal network of polar residues and water. Whereas $\mathrm{Asp}^{2.50}$ is replaced by Glu, the DRY motif is still present in these receptors. A sodium-independent mechanism of switching protonation states and rearranging the polar network is conceivable in these cases. However, future studies will be necessary to confirm if the polar signal transmission and activation mechanism we find is conserved in the limited number of atypical class A GPCRs.

\section{Materials and Methods.}

Defining Microswitch States. Residue side chain rotamer conformations were defined by in-house Gaussian style clustering to $\chi 1$ and $\chi 2$ dihedral angles, as seen in Fig. 7. Rotamer angles were determined every 240 ps using gmx_chi. The frame separation was chosen to be slightly smaller than the autocorrelation time of the conserved water dynamics with the fastest relaxation time (55). A Hanning window function was used to smooth the probability density function to remove noise and locate the state maxima for each rotamer conformation. Guess parameters for Gaussian fitting were then obtained by locating the full-width at half-maximum from each maximum and Gaussian curve fitting was applied. The microswitch conformational state limits were defined as the Gaussian intersects. Each discretized distribution was additionally checked visually to ensure the fitting was accurate.

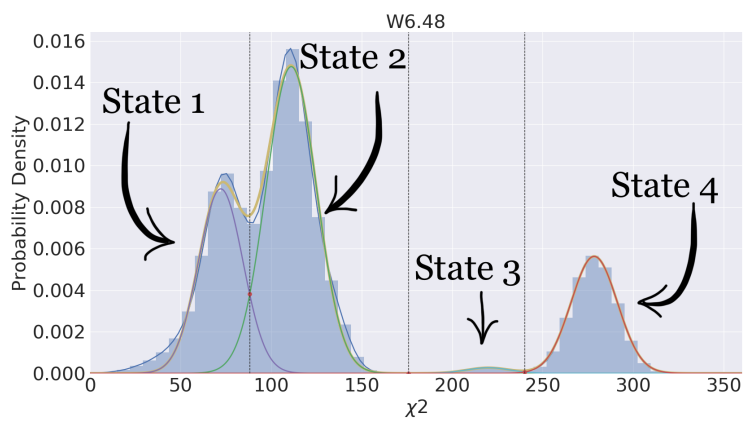

Fig. 7. Illustration of the Gaussian clustering method employed for defining discrete residue rotamer states. The example given is for the $\chi^{2}$ angle of the highly conserved tryptophan residue $\operatorname{Tr}^{6.48}$. State limits are defined as the intersects of each Gaussian and the maximum and minimum values of the angular distribution.

Water pocket states were defined by water molecule occupancy and using the water dipole moment to determine the molecular orientation. For $\mu \mathrm{OR}$, water pockets were defined using MDAnalysis (56) as spheres of radius $4 \AA$ centred on the geometric centres of (i) the tetrahedron formed by joining the C- $\alpha$ atoms of $\mathrm{Asn}^{1.50}-\mathrm{Asp}^{2.50}-\mathrm{Asn}^{7.49}-\mathrm{Leu}^{2.46}$; (ii) the 
triangle formed by joining the $\mathrm{C}-\alpha$ atoms of $\mathrm{Asn}^{7.45}-\mathrm{Asn}^{7.49}$ $\mathrm{Ala}^{6.38}$; (iii) the triangle formed by joining the $\mathrm{C}-\alpha$ atoms of Cys $^{6.47}$-Pro ${ }^{6.50}$-Cys ${ }^{7.37}$; and (iv,v) spheres of $5 \AA$ radius centred on the geometric centres of the tetrahedron formed by joining the $\mathrm{C}-\alpha$ atoms of $\mathrm{Asn}^{2.45}-\mathrm{Thr}^{3.42}-\mathrm{Val}^{4.45}-\mathrm{Trp}^{4.50}$, and the triangle $\mathrm{Val}^{3.48}-\mathrm{Arg}^{34.57}-\mathrm{Ala}^{4.42}$, respectively, as seen in Fig. 8. Similar positions were used for the $\delta \mathrm{OR}$ and $\mathrm{A}_{2 \mathrm{~A}} \mathrm{AR}$, determined by centering the water probability density in the geometric centre of the selected $\mathrm{C}-\alpha$ atoms.

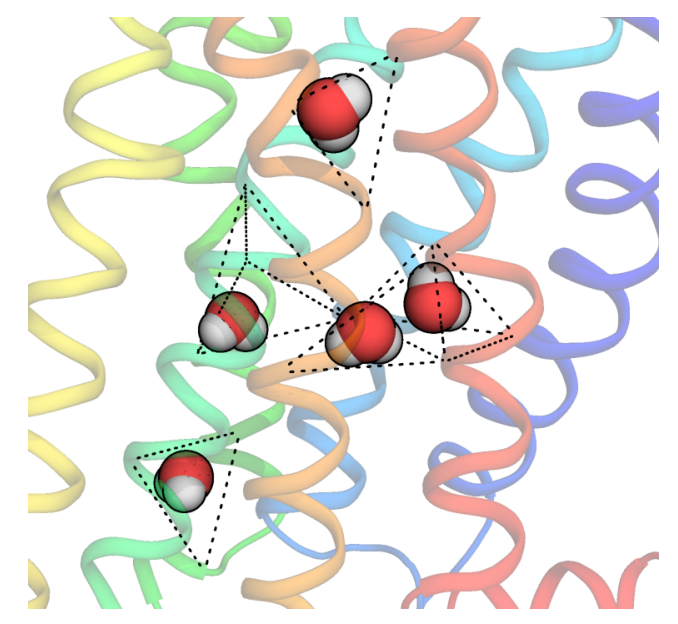

Fig. 8. Water pockets conserved in active and inactive crystal structures of $\mu$ OR, $\mathbf{A}_{2 \mathrm{~A}} \mathbf{A R}$ and $\delta \mathbf{O R}$. Pockets are defined as spheres of radii $4 \AA$ and $5 \AA$ centred on the centre of geometry of the respective triangles and tetrahedrons.

When water was not found in a specific water pocket at a certain time point, that pocket was assigned a discrete empty state. Water pockets must have all three water atoms within the pocket as defined above to be considered occupied. When water was found inside a pocket, the dipole moment vector for the water molecule $(\vec{\mu})$ was calculated by $\vec{\mu}=0.5\left(H_{1}+H_{2}\right)-O$, where $H_{i}$ is the $(\mathrm{x}, \mathrm{y}, \mathrm{z})$ coordinate of the $i$ th hydrogen, and $O$ is the (x,y,z) coordinate of the oxygen. The orientation of water molecules was then calculated by converting the dot product of the dipole vector and the simulation box axes vector into spherical coordinates, $\psi=\arctan \left(\frac{y}{x}\right)$ and $\theta=\arctan \frac{\sqrt{x^{2}+y^{2}}}{z}$, followed by the same Gaussian style clustering algorithm. Together, this allowed us to deduce the functional effect of each water pocket by combining the continuous distributions of water polarisation and the binary/discrete distributions of water pocket occupancy (i.e. occupied vs. unoccupied) into a univariate distribution.

Information Theory. Unlike cartesian correlation approaches, a state based mutual information approach resolves slow modes in protein dynamics that have the greatest functional relevance, reducing noise levels $(27,39)$. Causality can be inferred from our state based calculation of mutual information because the ion binding states in each ensemble are constrained (i.e. they are constant for each simulation), only changing state upon concatenation, and therefore the root change occurs at the ion binding site. The information transfer resolved therefore travels from the ion binding site to residues and internal waters. However, since SSI is symmet- ric in principle, note that the identified information pathway could also serve to support information flow towards the ion binding site.

The conformational state entropy (Shannon entropy) of microswitch $X$ was determined from $H(X)=-\sum_{i} p(i) \log _{2} p(i)$, where $p(i)$ is the probability of state $i$ occurring, defined using our definition of discrete states (Fig. 7). $H(X)$ is also a measure of the maximum information a microswitch can store (26). A regular average was taken across all common Ballesteros-Weinstein positions in the three receptors to deduce the average entropy of a specific residue. We used the state limits of the concatenated simulations for the conformational state entropy to extrapolate conclusions about the SSI transfer, thereby identifying the whether a microswitch is prohibited from communicating in a specific simulation state. Therefore, residues have zero entropy only if they occupy one of the information transfer states, and not just a single Gaussian distribution that spanned two information states.

SSI (mutual state information) between two microswitches $X$ and $Y$ was calculated using $S S I(X, Y)=H(X)+H(Y)-$ $H(X, Y)$, where $H(X, Y)$ is the joint entropy, quantifying the entropy of individual microswitches $X$ and $Y$ as one multivariate microswitch using our definition of discrete states (26).

The co-SSI (or interaction information) was calculated using

$$
\begin{array}{r}
\operatorname{coSSI}\left(X, Y, H_{2} \mathrm{O}\right)=S S I(X, Y)-S S I\left(X, Y \mid H_{2} O\right) \\
=H(X)+H(Y)+H\left(H_{2} O\right) \\
-H(X, Y)-H\left(X, H_{2} O\right)-H\left(Y, H_{2} O\right) \\
+H\left(X, Y, H_{2} O\right)
\end{array}
$$

where $\mathrm{SSI}\left(\mathrm{X}, \mathrm{Y} \mid \mathrm{H}_{2} \mathrm{O}\right)$ determines what magnitude of SSI shared between $X$ and $Y$ is dependent on the state of a specific third component, often an intercalating water molecule $\left(\mathrm{H}_{2} \mathrm{O}\right)$ (29). There are different interpretations of negative interaction information values in the literature (30-32). We adopted the interpretation used by LeVine \& Weinstein, in which negative interaction information serves to attenuate information transfer between two residues (31). We deemed SSI and co-SSI values of less than 0.05 bits insignificant on a 95\% significance level. As the changes made to the state of the ion binding site were binary (i.e. 1 bit of information - sodium-bound to sodium-free state; and sodium-free to $/ \mathrm{Asp}^{2.50}$-protonated state), the theoretical upper limit for all SSI and co-SSI values was therefore 1 bit.

To focus on information transfer pathways conserved in all three receptors, we determined the geometric mean of SSI and co-SSI from all common residue Ballesteros-Weinstein positions.

Protein structure preparations. All crystal structures $(\mu \mathrm{OR}$ pdb:4dkl, $\delta \mathrm{OR}$ - pdb:4n6h, $\mathrm{A}_{2 \mathrm{~A}} \mathrm{AR}$ - pdb:5olz) were obtained from GPCRdb (57), selecting the GPCRdb refined structure in which mutations are reversed to the canonical sequence and cleaved loops are re-modelled using MODELLER 9.18 to produce the final structure $(58,59)$. All transmembrane 
bioRxiv preprint doi: https://doi.org/10.1101/2020.08.28.271510; this version posted January 5, 2021. The copyright holder for this preprint (which was not certified by peer review) is the author/funder, who has granted bioRxiv a license to display the preprint in perpetuity. It is made available under aCC-BY 4.0 International license.

crystalline waters and ligands were kept in the structure, removing all external waters and non-protein molecules. The proteins were truncated and capped with acetyl and methyl groups at corresponding $\mathrm{N}$ and $\mathrm{C}$ terminals using PyMOL (60). As the $\mu \mathrm{OR}$ crystal structure did not resolve a sodium ion in the pocket, the sodium-bound structure was obtained by aligning the $\delta \mathrm{OR}$ crystal structure and placing the sodium ion in an identical position. Asp ${ }^{2.50}$-charged protein structures were prepared by removing sodium from the crystal structure and ensuring no sodium ion re-associated during the course of the simulation. The third, protonated-Asp ${ }^{2.50}$ protein state was prepared in the molecular dynamics software GROMACS-5.1.1 (61). Ligand structures were taken from their respective protein pdb files, and parameterised for molecular dynamics simulations in GROMACS-5.1.1 using ACPYPE (62).

MD simulations. We modelled the protein, membrane, and ligand using the amber99sb-ildn forcefield (63) in GROMACS-5.1.1 with virtual sites to allow a time-step of 4 fs. The proteins were embedded in a pre-equilibrated SLipid POPC membrane (64) using InflateGRO (65). The proteinmembrane complexes were solvated with a neutral solution of TIP3P water molecules (66) containing $\mathrm{NaCl}$ at $\sim 150$ $\mathrm{mM}$ concentration, with a box size of $\sim 128 \times 134 \times 125 \AA^{3}$. The systems were equilibrated in both the NVT and NPT ensembles at $310 \mathrm{~K}$ for $3 \mathrm{~ns}$ with position restraints on the ligand-receptor complex heavy atoms, with a further $70 \mathrm{~ns}$ of production run simulation considered as additional NPT equilibration. Following equilibration, simulations were performed for $1.7 \mu \mathrm{s}$ each at a constant temperature of $310 \mathrm{~K}$ and pressure of $1 \mathrm{bar}$, with the protein-ligand complex, membrane, and solution independently coupled to a temperature bath using a Nosé-Hoover thermostat with a time constant of 0.5 ps and a semi-isotropic Parrinello-Rahman barostat with a time constant of $5 \mathrm{ps}$ (67). The trajectory of the $\delta$ OR protonated at $\mathrm{Asp}^{2.50}$ was obtained from a previous simulation study $(53,68)$. All protein and lipid bond lengths were constrained with the LINCS algorithm (69), while water bond lengths were constrained using SETTLE (70).

\section{SUPPLEMENTARY MATERIALS}

\section{Fig. S1: Asp ${ }^{2.50}$-protonation SSI.}

Fig. S2: Sodium Expulsion SSI.

Fig. S3: Inter-hydroxyl distance between Tyr5.58-Tyr7.53 of $\mu \mathrm{OR}$

Fig. S4: Activation coordinate (TM2-TM6) in the $\delta \mathrm{OR}$.

Fig. S5: Asp. 2.50 -protonation co-SSI For Wat1.

Fig. S6: Asp ${ }^{2.50}$-protonation co-SSI For Wat2.

Fig. S7: Asp ${ }^{2.50}$-protonation co-SSI For Wat3.

Fig. S8: Asp 2.50 -protonation co-SSI For Wat4.

Fig. S9: Asp ${ }^{2.50}$-protonation co-SSI For Wat5.

Fig. S10: Sodium Expulsion co-SSI For Wat1.

Fig. S11: Sodium Expulsion co-SSI For Wat2.

Fig. S12: Sodium Expulsion co-SSI For Wat3.

Fig. S13: Sodium Expulsion co-SSI For Wat4.

Fig. S14: Sodium Expulsion co-SSI For Wat5.

\section{Bibliography}

1. Vsevolod Katritch, Vadim Cherezov, and Raymond C Stevens. Structure-Function of the G Protein-Coupled Receptor Superfamily. Annual Review of Pharmacology and Toxicology, 53(1):531-556, 2013. doi: 10.1146/annurev-pharmtox-032112-135923.

2. Alexander S Hauser, Sreenivas Chavali, Ikuo Masuho, Leonie J Jahn, Kirill A Martemyanov, David E Gloriam, and M Madan Babu. Pharmacogenomics of GPCR Drug Targets. Cell, 172(1):41 - 54.e19, 2018. ISSN 0092-8674. doi: https://doi.org/10.1016/j.cell.2017.11.033.
3. Vsevolod Katritch, Gustavo Fenalti, Enrique E Abola, Bryan L Roth, Vadim Cherezov, and Raymond C Stevens. Allosteric sodium in class A GPCR signaling. Trends in Biochemical Sciences, 39(5):233-244, 2014. ISSN 0968-0004. doi: https://doi.org/10.1016/j.tibs.2014. 03.002 .

4. Barbara Zarzycka, Saheem A. Zaidi, Bryan L. Roth, and Vsevolod Katritch. Harnessing ionbinding sites for GPCR pharmacology. Pharmacological Reviews, 71(4):571-595, 2019. ISSN 15210081. doi: 10.1124/pr.119.017863.

5. Vignir Isberg, Chris de Graaf, Andrea Bortolato, Vadim Cherezov, Vsevolod Katritch, Fiona H Marshall, Stefan Mordalski, Jean-Philippe Pin, Raymond C Stevens, Gerrit Vriend, and David E Gloriam. Generic GPCR residue numbers - aligning topology maps while minding the gaps. Trends in Pharmacological Sciences, 36(1):22-31, 2015. ISSN 01656147. doi: https://doi.org/10.1016/j.tips.2014.11.001.

6. Yinglong Miao, Alisha D Caliman, and J Andrew McCammon. Allosteric effects of sodium ion binding on activation of the $\mathrm{m} 3$ muscarinic g-protein-coupled receptor. Biophysical journal, 108(7):1796-1806, apr 2015. ISSN 1542-0086. doi: 10.1016/j.bpj.2015.03.003.

7. Libin Ye, Chris Neale, Adnan Sljoka, Brent Lyda, Dmitry Pichugin, Nobuyuki Tsuchimura, Sacha T Larda, Régis Pomès, Angel E García, Oliver P Ernst, et al. Mechanistic insights into allosteric regulation of the a $2 \mathrm{a}$ adenosine $\mathrm{g}$ protein-coupled receptor by physiological cations. Nature communications, 9(1):1-13, 2018.

8. Kate L. White, Matthew T. Eddy, Zhan Guo Gao, Gye Won Han, Tiffany Lian, Alexander Deary, Nilkanth Patel, Kenneth A. Jacobson, Vsevolod Katritch, and Raymond C. Stevens. Structural Connection between Activation Microswitch and Allosteric Sodium Site in GPCR Signaling. Structure, 26(2):259-269.e5, 2018. ISSN 18784186. doi: 10.1016/j.str.2017.12. 013

9. Daria N Shalaeva, Dmitry A Cherepanov, Michael Y Galperin, Gert Vriend, and Armen Y Mulkidjanian. G protein-coupled receptors of class $A$ harness the energy of membrane potential to increase their sensitivity and selectivity. Biochimica et Biophysica Acta (BBA) - Biomembranes, 1861(12):183051, 2019. ISSN 0005-2736. doi: https://doi.org/10.1016/j. bbamem.2019.183051

10. Xiaohu Hu, Yibo Wang, Amanda Hunkele, Davide Provasi, Gavril W Pasternak, and Marta Filizola. Kinetic and thermodynamic insights into sodium ion translocation through the $\mu$ opioid receptor from molecular dynamics and machine learning analysis. PLOS Computational Biology, 15(1):1-19, 2019. ISSN 15537358. doi: 10.1371/journal.pcbi.1006689.

11. Anirudh Ranganathan, Ron O Dror, and Jens Carlsson. Insights into the Role of Asp792.50 in $\beta 2$ Adrenergic Receptor Activation from Molecular Dynamics Simulations. Biochemistry, 53(46):7283-7296, nov 2014. ISSN 0006-2960. doi: 10.1021/bi5008723.

12. Owen N Vickery, Catarina A Carvalheda, Saheem A Zaidi, Andrei V Pisliakov, Vsevolod Katritch, and Ulrich Zachariae. Intracellular Transfer of $\mathrm{Na}+$ in an Active-State G-ProteinCoupled Receptor. Structure, 26(1):171 - 180.e2, 2018. ISSN 0969-2126. doi: https: //doi.org/10.1016/j.str.2017.11.013.

13. A. J. Venkatakrishnan, Xavier Deupi, Guillaume Lebon, Franziska M. Heydenreich, Tilman Flock, Tamara Miljus, Santhanam Balaji, Michel Bouvier, Dmitry B. Veprintsev, Christopher G. Tate, Gebhard F.X. Schertler, and M. Madan Babu. Diverse activation pathways in class A GPCRs converge near the G-protein-coupling region. Nature, 536:484-487, 2016. doi: 10.1038/nature 19107 .

14. A J Venkatakrishnan, Anthony $\mathrm{K} \mathrm{Ma}$, Rasmus Fonseca, Naomi R Latorraca, Brendan Kelly, Robin M Betz, Chaitanya Asawa, Brian K Kobilka, and Ron O Dror. Diverse GPCRs exhibit conserved water networks for stabilization and activation. Proceedings of the National Academy of Sciences, 116(8):3288-3293, 2019. ISSN 0027-8424. doi: 10.1073/pnas.1809251116.

15. Shuguang Yuan, Slawomir Filipek, Krzysztof Palczewski, and Horst Vogel. Activation of Gprotein-coupled receptors correlates with the formation of a continuous internal water pathway. Nature Communications, 5(4733), 2014. ISSN 20411723. doi: 10.1038/ncomms5733.

16. Vsevolod V Gurevich and Eugenia V Gurevich. Molecular Mechanisms of GPCR Signaling: A Structural Perspective. International journal of molecular sciences, 18(12), nov 2017. ISSN 1422-0067. doi: 10.3390/ijms 18122519.

17. Leonardo Pardo, Xavier Deupi, Nicole Dölker, María Luz López-Rodríguez, and Mercedes Campillo. The Role of Internal Water Molecules in the Structure and Function of the Rhodopsin Family of G Protein-Coupled Receptors. ChemBioChem, 8(1):19-24, jan 2007. ISSN 1439-4227. doi: 10.1002/cbic.200600429.

18. Yoonji Lee, Sun Choi, and Changbong Hyeon. Mapping the intramolecular signal transduction of G-protein coupled receptors. Proteins: Structure, Function and Bioinformatics, 82 (5):727-743, 2014. ISSN 10970134. doi: 10.1002/prot.24451.

19. Yoonji Lee, Sun Choi, and Changbong Hyeon. Communication over the Network of Binary Switches Regulates the Activation of A2A Adenosine Receptor. PLoS Computational Biology, 11(2):1-21, 2015. ISSN 15537358. doi: 10.1371/journal.pcbi.1004044.

20. B Trzaskowski, D Latek, S Yuan, U Ghoshdastider, A Debinski, and S Filipek. Action of molecular switches in GPCRs-theoretical and experimental studies. Current medicinal chemistry, 19(8):1090-1109, 2012. ISSN 1875-533X. doi: 10.2174/092986712799320556.

21. Katsufumi Tomobe, Eiji Yamamoto, Kholmirzo Kholmurodov, and Kenji Yasuoka. Wate permeation through the internal water pathway in activated GPCR rhodopsin. PLOS ONE, 12(5):e0176876, 2017. ISSN 19326203. doi: 10.1371/journal.pone.0176876.

22. Qingtong Zhou, Dehua Yang, Meng Wu, Yu Guo, Wanjing Guo, Li Zhong, Xiaoging Cai, Antao Dai, Wonjo Jang, Eugene Shakhnovich, Zhi Jie Liu, Raymond C. Stevens, Nevin A. Lambert, M. Madan Babu, Ming Wei Wang, and Suwen Zhao. Common activation mechanism of class a GPCRs. eLife, 8(e50279), 2019. ISSN 2050084X. doi: 10.7554/eLife.50279.

23. Oliver Fleetwood, Pierre Matricon, Jens Carlsson, and Lucie Delemotte. Energy landscapes reveal agonist control of g protein-coupled receptor activation via microswitches. Biochemistry, 59(7):880-891, 2020

24. Daniel G. Isom and Henrik G. Dohlman. Buried ionizable networks are an ancient hallmark of $\mathrm{G}$ protein-coupled receptor activation. Proceedings of the National Academy of Sciences of the United States of America, 2015. ISSN 10916490. doi: 10.1073/pnas.1417888112.

25. I. Rodriguez-Espigares, M. Torrens-Fontanals, J.K.S. Tiemann, et al. Gpcrmd uncovers the dynamics of the 3d-gpcrome. Nature Methods, 2020. doi: 10.1038/s41592-020-0884-y.

26. C E Shannon. A Mathematical Theory of Communication. Bell System Technical Journal, 27(3):379-423, 1948. doi: 10.1002/j.1538-7305.1948.tb01338.x. 
bioRxiv preprint doi: https://doi.org/10.1101/2020.08.28.271510; this version posted January 5, 2021. The copyright holder for this preprint (which was not certified by peer review) is the author/funder, who has granted bioRxiv a license to display the preprint in perpetuity. It is made available under aCC-BY 4.0 International license.

27. Nagarajan Vaidehi and Supriyo Bhattacharya. Allosteric communication pipelines in Gprotein-coupled receptors. Current Opinion in Pharmacology, 30:76-83, 2016. ISSN 14714892. doi: https://doi.org/10.1016/j.coph.2016.07.010.

28. Giovanni B Brandani, Marieke Schor, Cait E MacPhee, Helmut Grubmüller, Ulrich Zachariae, and Davide Marenduzzo. Quantifying disorder through conditional entropy: an application to fluid mixing. PloS one, 8(6):e65617, 2013.

29. W. McGill. Multivariate information transmission. Transactions of the IRE Professional Group on Information Theory, 4(4):93-111, 1954.

30. Anthony J Bell. The co-information lattice. In Proceedings of the Fifth International Work shop on Independent Component Analysis and Blind Signal Separation: ICA, volume 2003. Citeseer, 2003.

31. Michael V LeVine and Harel Weinstein. NbIT - A New Information Theory-Based Analysis of Allosteric Mechanisms Reveals Residues that Underlie Function in the Leucine Transporter LeuT. PLOS Computational Biology, 10(5):1-15, 2014. doi: 10.1371/journal.pcbi.1003603.

32. A Ghassami and N Kiyavash. Interaction information for causal inference: The case of directed triangle. In 2017 IEEE International Symposium on Information Theory (ISIT), pages 1326-1330, 2017. ISBN 2157-8117 VO -. doi: 10.1109/ISIT.2017.8006744.

33. Oliver $F$ Lange and Helmut Grubmüller. Generalized correlation for biomolecular dynamics. Proteins: Structure, Function, and Bioinformatics, 62(4):1053-1061, 2006. doi: 10.1002/ prot.20784.

34. Aysima Hacisuleyman and Burak Erman. Entropy Transfer between Residue Pairs and AlIostery in Proteins: Quantifying Allosteric Communication in Ubiquitin. PLOS Computational Biology, 13(1):1-23, 2017. doi: 10.1371/journal.pcbi.1005319.

35. Abhijeet Kapoor, Gerard Martinez-Rosell, Davide Provasi, Gianni de Fabritiis, and Marta Filizola. Dynamic and Kinetic Elements of $\mu$-Opioid Receptor Functional Selectivity. Scientific Reports, 7(1):11255, 2017. ISSN 2045-2322. doi: 10.1038/s41598-017-11483-8.

36. Nuray Sogunmez and Ebru Demet Akten. Distinctive communication networks in inactive states of $\beta 2$-adrenergic receptor: Mutual information and entropy transfer analysis. Proteins: Structure, Function and Bioinformatics, 2020. ISSN 10970134. doi 10.1002/prot. 25965

37. Kateri H. DuBay, Jacques P. Bothma, and Phillip L. Geissler. Long-range intra-protein communication can be transmitted by correlated side-chain fluctuations alone. PLOS Computational Biology, 2011. ISSN 1553734X. doi: 10.1371/journal.pcbi.1002168.

38. Christopher L McClendon, Gregory Friedland, David L Mobley, Homeira Amirkhani, and Matthew P Jacobson. Quantifying Correlations Between Allosteric Sites in Thermodynamic Ensembles. Journal of Chemical Theory and Computation, 5(9):2486-2502, sep 2009. ISSN 1549-9618. doi: 10.1021/ct9001812.

39. Sukrit Singh and Gregory R Bowman. Quantifying Allosteric Communication via Both Concerted Structural Changes and Conformational Disorder with CARDS. Journal of Chemical Theory and Computation, 13(4):1509-1517, apr 2017. ISSN 1549-9618. doi: 10.1021/acs.jctc.6b01181.

40. Supriyo Bhattacharya and Nagarajan Vaidehi. Differences in Allosteric Communication Pipelines in the Inactive and Active States of a GPCR. Biophysical Journal, 107(2):422434, 2014. ISSN 0006-3495. doi: https://doi.org/10.1016/j.bpj.2014.06.015.

41. Yuchen Zhou, Steven Ramsey, Davide Provasi, Amal El Daibani, Kevin Appourchaux, Soumen Chakraborty, Abhijeet Kapoor, Tao Che, Susruta Majumdar, and Marta Filizola. Predicted Mode of Binding to and Allosteric Modulation of the $\mu$-Opioid Receptor by Kratom's Alkaloids with Reported Antinociception In Vivo. Biochemistry, dec 2020. ISSN 0006-2960. doi: 10.1021/acs.biochem.0c00658.

42. Jop Briët and Peter Harremoës. Properties of classical and quantum Jensen-Shannon divergence. Physical Review A, 79(5):52311, may 2009. doi: 10.1103/PhysRevA.79.052311.

43. Martin Vögele. Pensa, December 2020.

44. X Edward Zhou, Karsten Melcher, and H Eric Xu. Structural biology of G protein-coupled receptor signaling complexes. Protein science : a publication of the Protein Society, 28(3): 487-501, mar 2019. ISSN 1469-896X. doi: 10.1002/pro.3526.

45. Manbir Sandhu, Anja M Touma, Matthew Dysthe, Fredrik Sadler, Sivaraj Sivaramakrish nan, and Nagarajan Vaidehi. Conformational plasticity of the intracellular cavity of GPCRGprotein complexes leads to G-protein promiscuity and selectivity. Proceedings of the $\mathrm{Na}$ tional Academy of Sciences, 116(24):11956 LP - 11965, jun 2019. doi: 10.1073/pnas. 1820944116.

46. Anne Steen, Olav Larsen, Stefanie Thiele, and Mette M Rosenkilde. Biased and G ProteinIndependent Signaling of Chemokine Receptors. Frontiers in Immunology, 5:277, 2014 ISSN 1664-3224. doi: 10.3389/fimmu.2014.00277.

47. Edward C Hulme. GPCR activation: a mutagenic spotlight on crystal structures. Trends in Pharmacological Sciences, 34(1):67-84, jan 2013. ISSN 0165-6147. doi: 10.1016/j.tips. 2012.11.002.

48. Jing Yu, Luis E Gimenez, Ciria C Hernandez, Yiran Wu, Ariel H Wein, Gye Won Han, Kyle McClary, Sanraj R Mittal, Kylie Burdsall, Benjamin Stauch, et al. Determination of the melanocortin-4 receptor structure identifies ca2+ as a cofactor for ligand binding. Science, 368(6489):428-433, 2020

49. Daniel G. Isom, Vishwajith Sridharan, Rachael Baker, Sarah T. Clement, David M. Smalley, and Henrik G. Dohlman. Protons as second messenger regulators of $G$ protein signaling. Molecular Cell, 2013. ISSN 10972765. doi: 10.1016/j.molcel.2013.07.012.

50. Xuejun $\mathrm{C}$ Zhang, Can $\mathrm{Cao}$, Ye Zhou, and Yan Zhao. Proton transfer-mediated GPCR activation. Protein \& Cell, 6(1):12-17, 2015. ISSN 1674-8018. doi: 10.1007/s13238-014-0106-4.

51. Mohana Mahalingam, Karina Martínez-Mayorga, Michael F Brown, and Reiner Vogel. Two protonation switches control rhodopsin activation in membranes. Proceedings of the National Academy of Sciences, 105(46):17795-17800, 2008. ISSN 0027-8424. doi: 10.1073/pnas.0804541105.

52. Udeep Chawla, Suchithranga Perera, Steven Fried, Anna Eitel, Blake Mertz, Nipuna Weerasinghe, Michael Pitman, Andrey Struts, and Michael F Brown. Activation of the GProtein-Coupled Receptor Rhodopsin by Water. Angewandte Chemie International Edition 2020. ISSN 1433-7851. doi: 10.1002/anie.202003342.

53. Owen N. Vickery, Jan-Philipp Machtens, Giulia Tamburrino, Daniel Seeliger, and Ulrich Zachariae. Structural Mechanisms of Voltage Sensing in G-Protein-Coupled Receptors. Structure, 24(6):997-1007, jun 2016. ISSN 0969-2126. doi: 10.1016/j.str.2016.04.007.
54. Jendrik Schöppe, Janosch Ehrenmann, Christoph Klenk, Prakash Rucktooa, Marco Schütz, Andrew S Doré, and Andreas Plückthun. Crystal structures of the human neurokinin 1 receptor in complex with clinically used antagonists. Nature communications, 10(1):1-11, 2019.

55. Yoonji Lee, Songmi Kim, Sun Choi, and Changbong Hyeon. Ultraslow Water-Mediated Transmembrane Interactions Regulate the Activation of A2A Adenosine Receptor. Biophysical journal, 111(6):1180-1191, sep 2016. ISSN 1542-0086. doi: 10.1016/j.bpj.2016.08.002.

56. Naveen Michaud-Agrawal, Elizabeth J Denning, Thomas B Woolf, and Oliver Beckstein. MDAnalysis: A toolkit for the analysis of molecular dynamics simulations. Journal of Computational Chemistry, 32(10):2319-2327, jul 2011. ISSN 0192-8651. doi: 10.1002/jcc.21787.

57. Vignir Isberg, Stefan Mordalski, Christian Munk, Krzysztof Rataj, Kasper Harpsøe, Alexander S. Hauser, Bas Vroling, Andrzej J. Bojarski, Gert Vriend, and David E. Gloriam. GPCRdb: An information system for G protein-coupled receptors. Nucleic Acids Research, 44(D1):D356-64, 2016. ISSN 13624962. doi: 10.1093/nar/gkv1178.

58. Benjamin Webb and Andrej Sali. Comparative Protein Structure Modeling Using MODELLER. Current Protocols in Bioinformatics, 54(1):5.6.1-5.6.37, 2016. doi: 10.1002/cpbi.3.

59. Gáspár Pándy-Szekeres, Christian Munk, Tsonko M Tsonkov, Stefan Mordalski, Kasper Harpsøe, Alexander S Hauser, Andrzej J Bojarski, and David E Gloriam. GPCRdb in 2018: adding GPCR structure models and ligands. Nucleic Acids Research, 46(D1):D440-D446, nov 2017. ISSN 0305-1048. doi: 10.1093/nar/gkx1109.

60. Warren L DeLano. Pymol: An open-source molecular graphics tool. CCP4 Newsletter on protein crystallography, 40(1):82-92, 2002.

61. Mark James Abraham, Teemu Murtola, Roland Schulz, Szilárd Páll, Jeremy C Smith, Berk Hess, and Erik Lindahl. GROMACS: High performance molecular simulations through multilevel parallelism from laptops to supercomputers. SoftwareX, 1-2:19-25, 2015. ISSN 23527110. doi: https://doi.org/10.1016/j.softx.2015.06.001.

62. Alan W Sousa da Silva and Wim F Vranken. ACPYPE - AnteChamber PYthon Parser interfacE. BMC Research Notes, 5(1):367, 2012. ISSN 1756-0500. doi: 10.1186/ 1756-0500-5-367.

63. Kresten Lindorff-Larsen, Stefano Piana, Kim Palmo, Paul Maragakis, John L Klepeis, Ron O Dror, and David E Shaw. Improved side-chain torsion potentials for the Amber ff99SB protein force field. Proteins, 78(8):1950-1958, jun 2010. ISSN 1097-0134. doi: 10.1002/prot.22711.

64. Joakim $P$ M Jämbeck and Alexander P Lyubartsev. An Extension and Further Validation of an All-Atomistic Force Field for Biological Membranes. Journal of Chemical Theory and Computation, 8(8):2938-2948, aug 2012. ISSN 1549-9618. doi: 10.1021/ct300342n.

65. Christian Kandt, Walter L Ash, and D Peter Tieleman. Setting up and running molecular dynamics simulations of membrane proteins. Methods, 41(4):475-488, 2007. ISSN 10462023. doi: https://doi.org/10.1016/j.ymeth.2006.08.006

66. William L Jorgensen, Jayaraman Chandrasekhar, Jeffry D Madura, Roger W Impey, and Michael L Klein. Comparison of simple potential functions for simulating liquid water. The Journal of Chemical Physics, 79(2):926-935, 1983. doi: 10.1063/1.445869.

67. Giovanni Bussi, Tatyana Zykova-Timan, and Michele Parrinello. Isothermal-isobaric molecular dynamics using stochastic velocity rescaling. The Journal of Chemical Physics, 130(7): 74101, feb 2009. ISSN 0021-9606. doi: 10.1063/1.3073889.

68. Owen N Vickery, Jan-Philipp Machtens, and Ulrich Zachariae. Membrane potentials regulating gpcrs: insights from experiments and molecular dynamics simulations. Current Opinion in Pharmacology, 30:44-50, 2016.

69. Berk Hess, Henk Bekker, Herman JC Berendsen, and Johannes GEM Fraaije. Lincs: a linear constraint solver for molecular simulations. Journal of computational chemistry, 18 (12):1463-1472, 1997.

70. Shuichi Miyamoto and Peter A Kollman. Settle: An analytical version of the shake and rattle algorithm for rigid water models. Journal of computational chemistry, 13(8):952-962, 1992.

\section{ACKNOWLEDGEMENTS}

We thank Seva Katritch, Andrei Pisliakov, and Martin Vögele for critical reading of and comments on the manuscript.

\section{FUNDING}

This work was supported by a BBSRC EASTBIO PhD studentship (to N.J.T.); a BBSRC Case award (to O.N.V.); and an MRC 4-year PhD studentship (to C.M.I.).

\section{AUTHOR CONTRIBUTIONS}

Conceptualization: N.J.T and U.Z. Methodology: N.J.T and U.Z. Formal analysis: N.J.T and U.Z. Investigation: N.J.T, O.N.V and U.Z. Writing (original draft): N.J.T and U.Z. Writing (review and editing): N.J.T, C.M.I and U.Z. Supervision: U.Z.

\section{COMPETING INTERESTS}

The authors declare that they have no competing interests. 